\title{
MEASUREMENT OF PROTON AND ANTI-PROTON INTENSITIES IN THE TEVATRON COLLIDER*
}

\author{
S. Pordes, W. Blokland ${ }^{\dagger}$ J. Crisp, B. Fellenz, R. Flora, A. Hahn, \\ T. S. Meyer, A. Tollestrup, FNAL, Batavia, IL 60510, USA
}

\begin{abstract}
This paper describes the techniques used to measure the intensities of the proton $(p)$ and anti-proton $(\bar{p})$ beams in the Tevatron collider. The systems provide simultaneous measurements of the intensity of the 36 proton and 36 antiproton bunches and their longitudinal profiles.
\end{abstract}

\section{INTRODUCTION}

Accurate measurement of the intensity of the proton and the anti-proton beams is a basic requirement of the Tevatron instrumentation. The proton and anti-proton fluxes enter directly into a calculation of the luminosity provided to the experiments and the life-times of the different beams can give information on the mechanisms for beam loss. Since the proton and anti-proton fluxes enter into the luminosity with equal status, it is desirable to measure them with comparable relative precision even though the antiproton intensity is typically only $10 \%$ of the proton intensity. A DCCT[1] provides a measure of the total current circulating in the machine but does not distinguish between proton and anti-proton contributions to this current. The individual bunch intensities are measured using a high bandwidth Resistive Wall-Current Monitor installed in the F11 section of the Tevatron where the arrival time of proton and anti-proton bunches is separated by $200 \mathrm{~ns}$.

\section{BASIC TECHNIQUE}

The design and construction of the Resistive WallCurrent Monitor and its connections (cabling) to the outside world are described in detail in ref[2]. The essential features of the RWCM for our purposes are

- the RWCM has a calculated resistance of 1.288 ohms;

- the RWCM is transformer coupled with a low end cutoff at about $3 \mathrm{kHz}$ and an upper limit at several $\mathrm{GHz}$;

- the RWCM is connected to the measuring electronics in the outside world by $320 \mathrm{~ns}$ of $7 / 8$ inch Andrews Heliax $^{T M}$ cable.

Figure 1 shows a schematic of the two measurement systems attached to the Resistive Wall Current Monitor. One system, the F(ast) B(unch) I(ntegrator), integrates the RWCM output during gates corresponding to the beam passage times; it presently takes two turns of the Tevatron to acquire its data, making 18 bunch measurements and taking a baseline sample in each abort gap (21 measurements)

\footnotetext{
* work performed under DOE contract no. DE-AC002-76CH03000

${ }^{\dagger}$ now at ORNL
}

each turn. Intensities are reported for both single-bucket and 5 bucket wide gates to give a measure of satellites around the main bunch. The other system, the S(ampled) $\mathrm{B}$ (unch) $\mathrm{D}$ (isplay), passes the RWCM signal to a $2 \mathrm{GHz}, 8$ bit sampling oscilloscope[3]. A measurement consists of two full turns of data (42,000 samples), one sweep triggered by the proton marker and one triggered by the antiproton marker: the resulting waveforms are analyzed to yield both the intensity and the longitudinal profile of each proton and anti-proton bunch. Because the anti-proton intensities are typically $10 \%$ or less of the proton intensities, both systems use separate channels for protons and antiprotons and set the anti-proton channel to have higher sensitivity; the FBI further uses separate electronics for the single and multiple bucket readings. The FBI can operate at a few hundred $\mathrm{Hz}$; the SBD returns bunch intensities and longitudinal profiles at about $0.5 \mathrm{~Hz}$, dominated by the oscilloscope readout time.

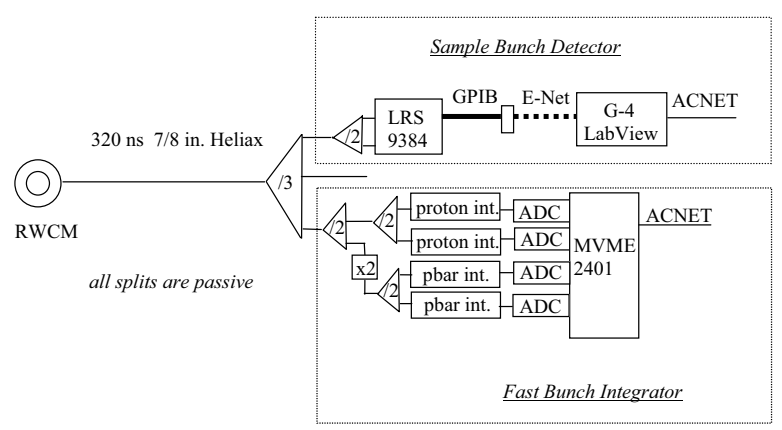

Figure 1: Schematic of FBI and SBD.

\section{ISSUES}

\section{Cable Effects}

Measurements of the cable[2] show an attenuation in $\mathrm{db}$ of $4.26 \sqrt{f / \mathrm{GHz}}$; this is consistent with the manufacturer data and a model where the frequency dependence is due to the skin effect. The accompanying dispersion of the RWCM signal along the $320 \mathrm{~ns}$ of cable is such that - almost independent of the beam shape - about $4.5 \%$ of the original signal arrives after the RF bucket containing the beam. The dispersion must also be accounted for when measuring the bunch-length.

\section{Baseline Effects}

Since the RWCM is transformer coupled, after a few turns, the net signal over a complete turn is zero. This 
means that the output baseline shifts. The shift is proportional to the beam intensity and can be estimated by noting that the protons (the dominant current) produce signals in 36 of the 1113 RF buckets; the average 'unoccupied' bucket will have a 'signal' of opposite sign and of magnitude $\sim 3 \%$ (36/1113) of the average proton bunch. For the protons, the baseline sampling thus produces an upward correction of about 3\%. For the anti-protons, which as mentioned, are typically $\sim 10 \%$ of the proton intensity, the baseline sampling is responsible for $\sim 30 \%$ of the reported $\bar{p}$ intensity.

\section{CALIBRATION}

There are two approaches available to calibrate the FBI and SBD - one is a quasi-absolute technique and one is based on comparison with the DCCT. The first involves taking the RWCM resistance as a given and applying a known pulse into the RWCM output cable to calibrate the FBI and SBD electronics. This is limited in principle by the accuracy of the pulser and requires transformer coupling and a proper simulation of the bunch structure and intensities to allow the baseline shift its full effect. Calibration using the DCCT as reference is based on the equation $D C C T=p_{\text {true }}+\bar{p}_{\text {true }}=p_{R W C M} \times\left(1+\frac{\bar{p}_{R W C M}}{p_{R W C M}}\right) \times C A L$ where $C A L$ is the calibration factor to be applied to the device looking at the RWCM signal. This approach requires no knowledge of the RWCM resistance and only the relative gains of the proton and anti-proton channels, not their absolute value. A small (few \%) correction does have to be made for satellites and any other out of the bucket beam all of which is seen by the DCCT but not by the SBD or FBI.

\section{$F$ (ast) B(unch) I(ntegrator)}

The FBI is used as a fast response system. The system calibration is harder to maintain in principle because 4 independent integrators are used to provide the single bucket, multi-bucket, proton and anti-proton measurements. The software to allow all measurements to be made on two integrators and to reduce the baseline issues by removing the amplifier in front of the anti-proton channel is in process. Figure 2 shows a comparison of the FBI and the SBD intensities recorded during a recent store. The plot is drawn such that the FBI and SBD should lie on top of each other - the agreement is quite good except for the anti-proton intensity at the beginning of the store. Examination of the FBI anti-proton baseline sample shows that it does not have the required proportionality to the total beam current probably because of some saturation in the electronics from the proton pulses and this may be responsible for the discrepancy. At present, the accuracy of the FBI is adequate for operations and we have concentrated on understanding the SBD as the device likely to give the more accurate measurement.

\section{The S(ampled) B(unch) D(isplay)}

Figure 3 shows one proton and one anti-proton bunch as recorded by the SBD. The darker trace is the proton chan-

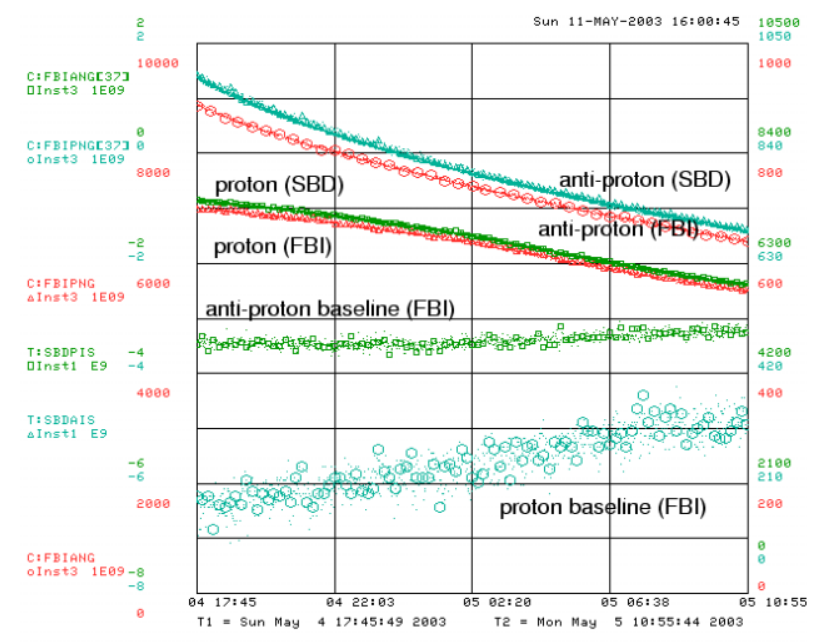

Figure 2: Comparison of FBI and SBD measurements

nel, the lighter trace is the anti-proton channel; the oscilloscope baseline is offset to be able to use the full dynamic range. The proton channel shows a small pulse (the antiproton bunch) on the left and the proton bunch at the right; the anti-proton channel shows the anti-proton bunch at the left and the proton bunch, which saturates, at the right. The time displacement between a given bunch as seen by the two channels is due to the timing difference of the proton and anti-proton markers. The satellites on the proton bunch are clearly visible in the anti-proton channel, as is the dispersion on the tail.

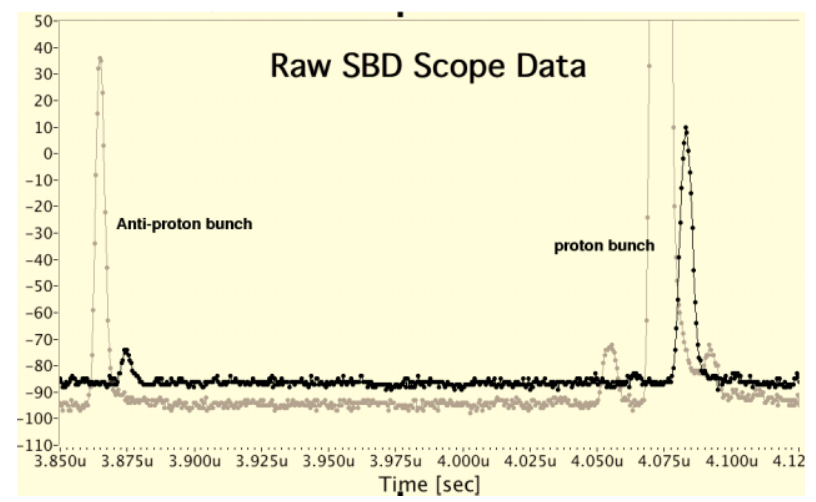

Figure 3: An SBD display of one proton and one antiproton bunch

\section{SBD Baseline Analysis}

The intensity reported by the SBD is quite sensitive to the baseline determination - an error of 1 count in the baseline is about $4 \%$ in intensity. After some experimentation, a histogramming technique has been adopted where the data from a sweep are histogrammed and a parabola is fit through the most common datum and its neighbors on either side. A typical data set is shown in figure 4.

Unlike algorithms based on sampling parts of the turn where no beam is expected, this process is robust at all 


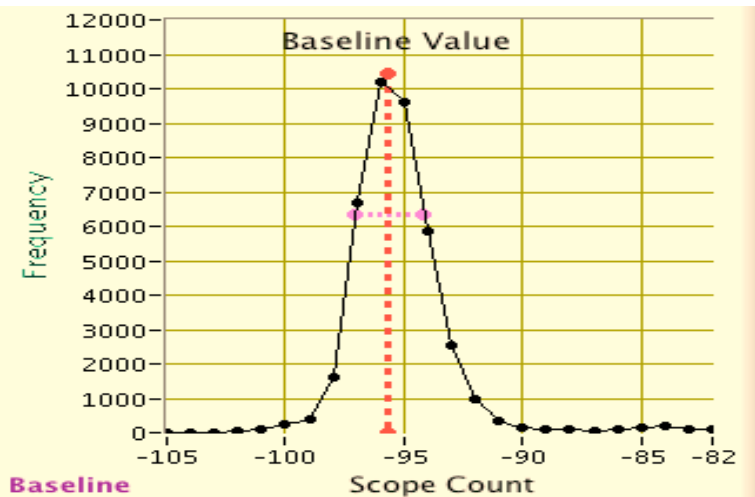

Figure 4: A baseline histogram showing the peak (the baseline) and the effective input noise

times including during cogging operations and the values it produces are good to a fraction of a count. The dispersive tail from the proton pulses tends to give a baseline which is slightly too high on the anti-proton channel; this contributes an $\sim 1 \%$ underestimate of the $\bar{p}$ intensity. A larger correction is due to the effect of the dispersion shifting part of the signal outside the RF bucket (see figure 5).

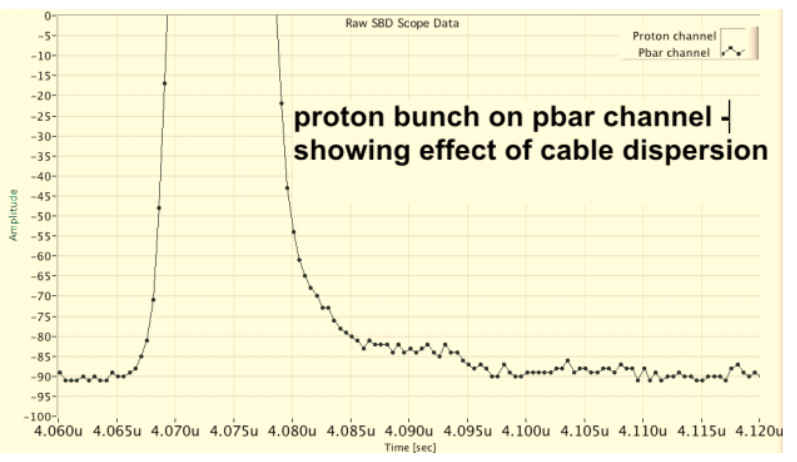

Figure 5: A proton bunch as seen on the anti-proton channel; note the dispersion on the trailing edge

\section{Dispersion Compensation}

The SBD was originally designed to provide a measurement of the longitudinal profile and while the effect of the dispersion on the intensity measurement could be corrected by a fudge factor, there is considerable interest in recovering the true input signal. The transfer function of the cable is linear and causal which means that (within the bandwidth of the measurement) it is in principle possible to recover the original signal provided one knows the transfer function. A compensating software filter has been developed based on the cable model - for details see ref[4] - and is applied to the data read from the scope. The filter as currently deployed has 77 taps corresponding to two RF buckets sampled at $2 \mathrm{GHz}$ and is applied to each input datum (starting from the 78th). Figure 6 shows the FIR filter - 'now' is at the right.

The form of the FIR can be understood in that any portion of the output at a given time is due to the signal at that time plus some fraction of earlier components of the signal

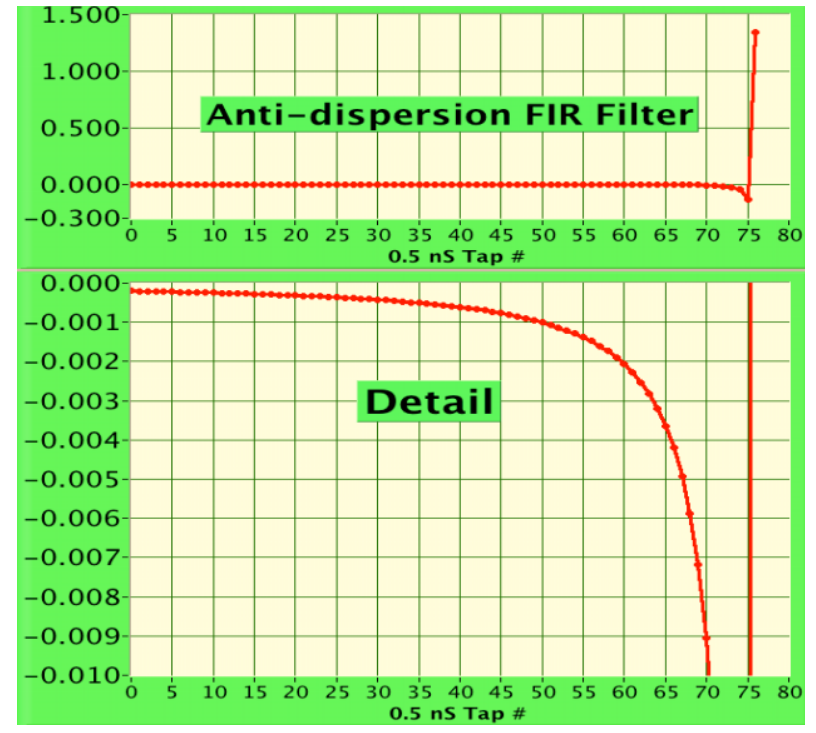

Figure 6: The dispersion compensation filter.

which have dispersed into the present; the FIR enhances the immediate portion and subtracts appropriate fractions of the earlier components. Figure 7 shows the effect on an anti-proton bunch. The performance is quite satisfactory.

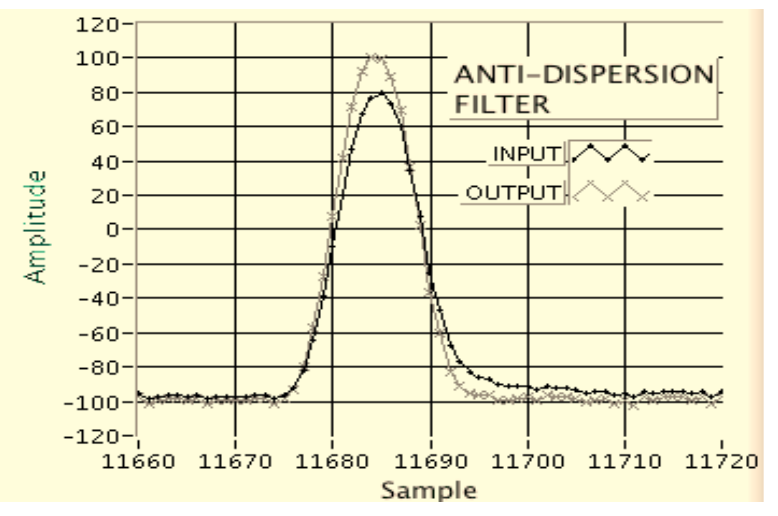

Figure 7: A bunch before and after the FIR filter

\section{SUMMARY}

Two systems are available at the Tevatron to measure individual proton and anti-proton bunch intensities with an accuracy of a few percent. A software FIR filter to compensate for cable dispersion has been implemented in the $2 \mathrm{GHz}$ sampling system and recovers the input signal for both intensity and longitudinal profile measurements.

\section{REFERENCES}

[1] R.C. Webber, AIP Conf. Proc. 333, Beam Inst. Workshop, Vancouver, 3-23 (1994).

[2] J. Crisp and B. Fellenz, Fermilab TM-2208, 2003

[3] Lecroy Research Systems, 9384

[4] A. Tollestrup, FNAL Beams Document Database Note 601 (2003). 